\title{
PRESERVERS OF PSEUDO SPECTRA OF OPERATOR JORDAN TRIPLE PRODUCTS
}

\author{
M. Bendaoud, A. Benyouness And M. SARIH
}

Abstract. Let $\mathscr{H}$ be an infinite-dimensional complex Hilbert space and let $\mathscr{L}(\mathscr{H})$ be the algebra of all bounded linear operators on $\mathscr{H}$. For $\varepsilon>0$ and $T \in \mathscr{L}(\mathscr{H})$, let $r_{\varepsilon}(T)$ denote the $\varepsilon$-pseudo spectral radius of $T$. We characterize surjective maps $\phi$ on $\mathscr{L}(\mathscr{H})$ which satisfy

$$
r_{\varepsilon}(\phi(T) \phi(S) \phi(T))=r_{\varepsilon}(T S T)
$$

for all $T, S \in \mathscr{L}(\mathscr{H})$. As application, mappings from $\mathscr{L}(X)$ onto itself that preserve the pseudo spectrum of Jordan triple product of operators are described. We also obtain analogous results for the finite-dimensional case, without the surjectivity assumption on $\phi$.

Mathematics subject classification (2010): 47B49, 47A10, 47A25.

Keywords and phrases: Operator, pseudo spectrum, pseudo spectral radius, Jordan triple product, nonlinear preservers.

\section{REFERENCES}

[1] M. Bendaoud, Preservers of local spectrum of matrix Jordan triple products, Linear Algebra Appl. 471, 1 (2015), 604-614.

[2] M. Bendaoud, Preservers of local spectra of matrix sums, Linear Algebra Appl. 438, 5 (2013), $2500-2507$.

[3] M. Bendaoud, M. Douimi And M. SARIH, Maps on matrices preserving local spectra, Linear Multilinear Algebra 61, 7 (2013), 871-880.

[4] M. Bendaoud, M. JABBAR AND M. SARIH, Preservers of local spectra of operator products, Linear Multilinear Algebra 63, 4 (2015), 806-819.

[5] R. Bhatia, P. ŠEmrl And A. R. Sourour, Maps on matrices that preserve the spectral radius distance, Studia Math. 134, 1-3 (1999), 99-110.

[6] J. T. Chan, C. K. Li And N. S. SzE, Mappings preserving spectra of products of matrices, Proc. Amer. Math. Soc. 135, 4 (2007), 977-986.

[7] J. T. Chan, C. K. Li AND N. S. SzE, Mappings on matrices: invariance of functional values of matrix products, J. Austral. Math. Soc. 81, 2 (2006), 165-184.

[8] J. Cui, V. Forstall, C.-K. Li and V. Yannello, Properties and preservers of the pseudospectrum, Linear Algebra Appl. 436, 2 (2012), 316-325.

[9] J. CUI AND J. Hou, Maps leaving functional values of operator products invariant, Linear Algebra Appl. 428, 7 (2008), 1649-1663.

[10] J. Cui AND C.-K. LI, Maps preserving peripheral spectrum of Jordan products of operators, Oper. Matrices 6, 6 (2012), 129-146.

[11] J. Cui, C. K. Li AND Y. T. Poon, Pseudospectra of special operators and pseudospectrum preservers, J. Math. Anal. Appl. 419, 2 (2014), 1261-1273.

[12] J. Cui, C. K. Li And Y. T. Poon, Preservers of unitary similarity functions on Lie products of matrices, Linear Algebra Appl. (2015), doi:101016/j.laa.2015.02.036.

[13] G. Dobovišek, B. KuZma, G. LeŠnJak, C. K. Li And T. Petek, Mappings that preserve pairs of operators with zero triple Jordan product, Linear Algebra Appl. 426, 2-3 (2007), 255-279. 
[14] G. Dolinar, J. Hou, B. KuZMa AND X. QI, Spectrum nonincreasing maps on matrices, Linear Algebra Appl. 438, 8 (2013), 3504-3510.

[15] H. GAO, *-Jordan-triple multiplicative surjective maps on $B(H)$, J. Math. Anal. Appl. 401, 1 (2013), 397-403.

[16] J. C. Hou, C. K. LI AND N. C. Wong, Maps preserving the spectrum of generalized Jordan product of operators, Linear Algebra Appl. 432, 4 (2010), 1049-1069.

[17] G. K. KUMAR AND S. H. KULKARNI, Linear maps preserving pseudospectrum and condition spectrum, Banach J. Math. Anal. 6, 1 (2012), 45-60.

[18] L. Molnár, Some characterizations of the automorphisms of $B(H)$ and $C(H)$, Proc. Amer. Math. Soc. 130, 1 (2001), 111-120.

[19] L. N. Trefethen And M. Embree, Spectra and Pseudospectra, The Behavior of Nonormal Matrices and Operators, Princeton University Press, Princeton, 2005.

[20] P. B. YALE, Automorphism of the complex numbers, Math. Mag. 39, 2 (1966), 135-141.

[21] W. Zhang AND J. Hou, Maps preserving peripheral spectrum of Jordan semi-triple products of operators, Linear Algebra Appl. 435, 6 (2011), 1326-1335. 